FORMATION Formation emploi

Revue française de sciences sociales

143 | Juillet-Septembre 2018

Le retour en formation : une vraie chance?

\title{
Travailler sur la socialisation des jeunes non qualifiés dans un dispositif de seconde chance : le sens de leurs réticences
}

Working on the socialization of non-qualified youth in a high school program:

the sense of heckling

Zweiten Chance : der Grund ihrer Zurückhaltung

Trabajar sobre la socialización de los jóvenes sin calificación en un dispositivo de segunda oportunidad : el sentido de sus reticencias

Florian Asséré

\section{(2) OpenEdition}

Journals

\section{Édition électronique}

URL : http://journals.openedition.org/formationemploi/6214

DOI : 10.4000/formationemploi.6214

ISSN : 2107-0946

Éditeur

La Documentation française

Édition imprimée

Date de publication : 20 novembre 2018

Pagination : $99-118$

ISSN : 0759-6340

\section{Référence électronique}

Florian Asséré, «Travailler sur la socialisation des jeunes non qualifiés dans un dispositif de seconde chance : le sens de leurs réticences », Formation emploi [En ligne], 143 | Juillet-Septembre 2018, mis en ligne le 20 novembre 2020, consulté le 05 novembre 2020. URL : http://journals.openedition.org/

formationemploi/6214; DOI : https://doi.org/10.4000/formationemploi.6214 


\title{
Travailler sur la socialisation des jeunes non qualifiés dans un dispositif de seconde chance : le sens de leurs réticences
}

\author{
FLORIAN AsSÉRÉ \\ Doctorant et chargé de cours à l'université Paris VIII Vincennes-Saint-Denis, au laboratoire \\ CIRCEFT (Centre interdisciplinaire de recherche: culture, éducation, formation, travail) au sein de \\ l'équipe ESCOL (éducation-scolarisation)
}

Résumé

Travailler sur la socialisation des jeunes non qualifiés dans un dispositif de seconde chance : le sens de leurs réticences

Comment les jeunes non qualifiés réagissent-ils au travail de socialisation opéré par les dispositifs de seconde chance à destination des décrocheurs? Le dispositif étudié cherche à favoriser un travail sur soi de la part des jeunes accompagnés qui voient dans cette rupture avec l'enseignement traditionnel une preuve supplémentaire de leur stigmate. Ces derniers mettent notamment en place un chahut loin d'être anomique. La mise en perspective de ces dispositifs avec les changements intervenus dans les politiques sociales nous éclairera sur le sens de ces réactions.

Mots clés : socialisation, abandon des études, représentation de la formation, mesure jeune, accompagnement en formation, école de la deuxième chance, jeune en difficulté

Abstract

Working on the socialization of non-qualified youth in a high school program : the sense of heckling

How the non qualified youth react to the socialization work operated in high school dropout programs? The programme we are looking at focuses on encouraging the students to work upon themselves. For these dropout students, this cease with the traditional way of teaching, is another proof of their stigma. They notably establish a certain amount of heckling far from being anomic. Putting this programme into perspective with the evolution of french social policies will reveal the meaning of these behaviors.

Keywords: socialization, rop out, perception of trainin, youth employment scheme, coaching in training, second-chance school, young person in difficulty

Journal of Economic Literature: I 28

Traduction : Auteur. 
"Ça sert à rien cette classe. C'est quoi ça MLDS ? Vous croyez que je vais me lever le matin pour aller à la truc de décrochage scolaire je sais pas quoi? Moi je veux des vrais cours avec du vrai français, des vrais maths, du vrai anglais!» (Jonathan, élève dans une classe du dispositif MLDS - Mission de lutte contre le décrochage scolaire).

Dans une interview récente, l'ancienne ministre de l'Éducation nationale, Najat VallaudBelkacem, se félicitait des résultats de la politique menée contre le décrochage scolaire. La multiplication des structures d'aide au retour à la formation aurait notamment permis d'abaisser le taux de décrocheurs, chez les 18-25, ans en dessous du seuil des $10 \%$ préconisé par l'Union européenne ${ }^{1}$.

La notion de décrochage scolaire obtient un succès assez tardif, en France, et doit beaucoup aux préoccupations européennes sur le sujet. En 2000, le sommet de Lisbonne dresse un plan décennal dont trois des cinq objectifs principaux en matière d'éducation concernent de près ou de loin le décrochage scolaire (Bernard, 2011).

En 1999, le programme " Nouvelles Chances ", de l'Éducation nationale, s'intéresse aux ruptures dans les parcours de qualification. Il mettra en place La Mission générale d'insertion (MGI), aujourd'hui devenue Mission de lutte contre le décrochage scolaire (MLDS). Créée en 2013, la MLDS poursuit deux objectifs, à savoir réduire le nombre de sorties du système éducatif sans diplôme et prendre en charge les jeunes non diplômés âgés de 16 ans et plus, afin de leur faciliter l'accès à une formation professionnalisante.

Cet accompagnement peut passer, soit par l'inscription dans une "classe MLDS " proposant un parcours dit de "remobilisation ", adapté aux jeunes ayant interrompu leur scolarité, soit par un suivi individualisé grâce à une plateforme de soutien et d'appui aux décrocheurs (PSAD) regroupant, à l'échelle d'un district, coordinateurs du dispositif, assistantes sociales, associations locales, éducateurs, élus et parfois entrepreneurs. Les classes MLDS sont généralement situées dans des lycées professionnels ou polyvalents. Au nombre de 70 dans l'académie de Créteil, les classes MLDS ont accueilli environ 1800 jeunes durant l'année scolaire 2017-2018². Chaque classe accueille de 25 à 30 jeunes et l'effectif d'une classe peut varier en raison des entrées et des sorties du dispositif au cours de l'annéés.

1. Entretien paru le 3 mars 2017 dans Vousnousils, un magazine en ligne spécialisé dans l'éducation.

2. Notons néanmoins qu'une minorité de ces 70 classes a d'autres fonctions, comme l'accueil d'élèves étrangers ou d'élèves ayant raté à plusieurs reprises leur baccalauréat. Concernant les plateformes d'appui et de soutien aux décrocheurs, il en existe 30 dans l'académie et 380 en France, mais il est difficile de dénombrer les jeunes suivis. De plus, ce suivi est très hétérogène, allant du simple appel téléphonique à un suivi de proximité sur plusieurs années.

3. En effet, certains jeunes se retrouvent dans le dispositif suite à une exclusion d'établissement ou à un décrochage en cours d'année. À L'inverse, d'autres peuvent obtenir une réintégration dans une filière ordinaire au second, voire au troisième trimestre. 
Les coordinateurs des classes MLDS sont chargés de nombreuses tâches, allant du recrutement des enseignants à celui des élèves, en passant par la création d'un emploi du temps cohérent et stable. Ils sont parfois secondés par d'autres membres du personnel, comme les conseillers principaux d'éducation (CPE), les auxiliaires de vie scolaire (AVS) ou les agents administratifs.

Les classes accueillent un ensemble assez hétéroclite d'élèves, certains n'ayant pas trouvé d'affectation dans un établissement, d'autres ayant été exclus, rappelés par les établissements grâce au logiciel de suivi des élèves ou encore aiguillés ici par un centre d'information et d'orientation (CIO), l'intermédiaire des missions locales, ou encore les assistantes sociales et éducateurs présents dans les plateformes de soutien et d'appui aux décrocheurs. Néanmoins, âgés dans les dispositifs étudiés, de 16 à 19 ans, les jeunes accompagnés ont en commun d'être très majoritairement issus de familles à faibles capitaux économiques et culturels ${ }^{4}$.

Notre corpus provisoire est constitué de 15 entretiens avec des acteurs impliqués dans deux dispositifs MLDS ( $\mathrm{N}=15)$, 11 entretiens informels avec d'anciens et actuels élèves de ces classes $(\mathrm{N}=11)$, d'observations de cours, ateliers et projets menés par différents acteurs $(\mathrm{N}=10)$, ainsi que d'une observation participante au sein d'un atelier de sociologie que nous avons mis en place dans une classe MLDS 5 . Des précisions sur le dispositif d'enquête et les choix méthodologiques sont détaillées dans l'encadré $\mathbf{1}$.

La lutte contre le décrochage scolaire implique aujourd'hui de nombreux dispositifs aux modalités très diverses (Blaya, 2010). Ces derniers peuvent être catégorisés selon leur logique coercitive ou incitative (Brunot, Saujat \& Félix, 2015). Représentée notamment par la loi Ciotti ${ }^{6}$, qui visait à lutter contre l'absentéisme en menaçant de supprimer les allocations familiales aux parents des enfants concernés, ou encore par des dispositifs mêlant action sociale et intervention policière, comme ceux de soutien à la parentalité décrits par Jessica Pothet (2016), cette première tendance des politiques de lutte contre le décrochage fait appel à "la main droite de l'État " $^{7}$. À l'inverse, le dispositif d'ampleur le plus récent, la MLDS, cherche à se définir, tant dans les textes régissant son action que dans les propos des acteurs de terrain, comme une action

$4.48 \%$ des décrocheurs ont un père ouvrier et seuls $5 \%$ un père cadre (INSEE, 2013). Cette origine sociale populaire est certainement plus importante sur nos deux terrains en raison des caractéristiques sociales des territoires concernés. Aucun des élèves avec lesquels nous nous sommes entretenus n’avait de parents exerçant des fonctions intermédiaires ou supérieures.

5. Les données et les réflexions de l'article sont issues d'un travail de thèse sous la direction de Stéphane Bonnéry, ainsi que d'un mémoire de Master 2 en sociologie encadré par Charles Soulié. Lauteur s'est également appuyé sur son expérience d'assistant pédagogique et d'enseignant vacataire au sein de l'établissement Jules Vallès.

6. La Loi Ciotti fut néanmoins abrogée le 17 janvier 2013, trois ans après avoir été votée.

7. Pierre Bourdieu oppose la main droite de l'État, avec ses fonctions régaliennes, à sa main gauche et son rôle d'État-providence (Bourdieu, 2012). 
incitative et bienveillante. Les plateformes d'appui et de soutien aux décrocheurs regroupent ainsi des membres de l'institution scolaire, des représentants d'associations locales et des assistantes sociales, en faisant appel à " la main gauche de l'État ».

Néanmoins, cette dernière a connu des évolutions majeures, aussi bien dans ses contenus que dans ses modes d'action et leurs effets. Aux droits et protections collectifs liés au travail dans un contexte de plein emploi, se sont substituées des politiques d'aides sociales individualisées et contractualisées qui tendent à stigmatiser les personnes aidées et à leur faire porter la responsabilité de leurs situations (Castel, 1995). Ce contexte d'évolution des politiques publiques nous éclairera sur le sens des réactions des jeunes face à certaines modalités d'un dispositif permettant d'appréhender ces politiques à l'échelle microsociale.

Nous nous demanderons alors, à travers une enquête de terrain menée au sein d'un dispositif de seconde chance donnée aux jeunes non qualifiés, ce que nous disent les réactions des individus accompagnés sur la nature de cette seconde chance. Pourquoi un dispositif de raccrochage fait-il l'objet d'une remise en cause collective de ceux qu'il est censé aider ? Comment comprendre l'hostilité à son égard ?

La première partie de cet article s'intéressera aux réactions des jeunes accompagnés face aux ambitions socialisatrices assumées du dispositif. Dans la seconde partie, le chahut ${ }^{8}$ ayant cours dans les dispositifs à l'encontre de certaines de ses modalités nous interrogera sur leurs effets et leurs similitudes avec les nouvelles politiques sociales.

\section{Encadré 1. La méthode d'enquête}

Les deux dispositifs étudiés concernent des lycées professionnels situés en banlieue parisienne, au sein de l'académie de Créteil. Les établissements comme les prénoms des enquêtés ont été anonymisés. La sélection des quinze personnes interviewées visait à obtenir un échantillon de toutes les catégories d'intervenants : coordinateurs du dispositif, enseignants, intervenants extérieurs menant des projets artistiques ou ateliers à visée professionnalisante, deux assistantes sociales, ainsi qu'une élue affectée à la question du décrochage scolaire dans la ville où est situé l'établissement Olympe de Gouges. Les observations en classe sont constituées d'une observation participante auprès de notre propre cours, d'une autre au sein d'un atelier courtmétrage pendant une année, d'observations ponctuelles parmi différents ateliers, de sorties scolaires, ainsi que de réunions de concertation.

8. Selon J. Testanière (1967, p.19), "il faut appeler chahut tout cas de désordre qui résulte directement ou indirectement d'un acte positif ou négatif, accompli par l'élève lui-même, et quil savait devoir produire ce résultat. " 
La majorité des discours des jeunes ont été collectés par le biais d'entretiens informels avec onze d'entre eux. Les jeunes accompagnés étant d'origines et d'âges divers, il existe peu d'interconnaissance entre eux. Bien qu'ils soient considérés comme décrocheurs, les causes de leur entrée dans le dispositif sont elles aussi hétérogènes : interruption d'une scolarité en seconde ou première, non affectation après la troisième, reprise d'études suite à une année sans scolarisation, exclusion d'un établissement, ancienne ou actuelle carrière délinquante poussant à une rescolarisation valorisée par la Protection judiciaire de la jeunesse (PJJ). Cohérent avec les statistiques nationales, notre échantillon a une répartition genrée plutôt à l'avantage des garçons, au nombre de six, et les enquêtés ont des parents ouvriers, employés ou au chômage. Ces entretiens ont pris le plus souvent la forme de nombreuses discussions en tête-à-tête et en petits groupes dans l'établissement, au détour d'un couloir, devant la grille, parfois accompagnés de surveillants, voire à l'extérieur.

Nous avons privilégié les entretiens informels pour trois raisons. D'une part, ils atténuent certains inconvénients de l'entretien directif ou semi-directif, attribuables à la distance sociale enquêteurs-enquêtés (Bruneteaux \& Lanzarini, 1998). En Effet, l'enquêteur pourrait se heurter à des discours stéréotypés, construits au long de toute une série d'interactions avec les travailleurs sociaux auxquels le sociologue pourrait être assimilé. Dans notre cas, ce discours stéréotypé présenterait plutôt les caractéristiques de celui exposé durant les nombreux entretiens individuels auxquels les jeunes accompagnés se retrouvent très régulièrement confrontés.

Par ailleurs, notre présence dans l'un des établissements accueillant le dispositif MLDS (Mission de lutte contre le décrochage scolaire), en tant qu'assistant pédagogique, notre âge relativement jeune et notre proximité avec les surveillants nous amenaient à recueillir de facto de nombreux discours de manière informelle. Le choix des entretiens informels exprimait alors autant un partipris théorique qu'un « choix de nécessité ».

Enfin, les entretiens informels nous permettaient d'avoir accès à des enquêtés qui ne se seraient pas forcément prêtés au jeu de l'entretien formel ou qui auraient une vision plutôt critique du dispositif.

Les entretiens informels ont aussi leurs inconvénients. Le principal est la perte d'informations importantes du fait d'une retranscription ex post. De même, les rencontres, parfois fortuites, imposent de multiplier les supports de prise de notes (carnet, ordinateur portable, feuille volante, smartphone, etc.)

Les entretiens informels ne sont pas exempts d'un travail méthodologique, bien au contraire. II s'agit, sans support écrit, de garder en tête les grandes lignes de ses questionnements dans des discussions presque ordinaires. Nous consignions par la suite les réponses et les autres éléments de discussion au sein d'un même fichier. Ce dernier nous permettait de détecter des oublis éventuels qui, comme les interruptions de discussion, nous imposaient parfois de nous entretenir à nouveau avec les enquêtés.

L'entretien informel suppose également de « bricoler » en permanence pour gérer des interactions instables qui s'appuient sur des formes ordinaires d'échanges (Bruneteaux \& Lanzarini, op. cit.). En l'occurrence, il était question pour nous d'essayer d'atténuer temporairement notre statut d'enseignant dans ce dispositif pour nous appuyer sur le statut moins défini et plus abordable de l'assistant pédagogique, tout en protégeant celui d'enseignant d'une trop grande familiarité qui aurait pu se répercuter sur l'image de l'enseignant et la gestion de classe. Sans pour autant céder aux « illusions de la neutralisation » de la situation d'enquête (Mauger, 1991), la spontanéité des propos recueillis dans ces conversations nous incitait à poursuivre dans cette voie. 


\section{Des réactions hostiles au travail de socialisation}

Le terme de dispositif est aujourd'hui abondamment utilisé dans l'institution scolaire pour qualifier des regroupements de nature très différente. Son usage désigne généralement des parties de l'institution centrées sur la résolution d'un problème particulier, relativement autonomes et adaptables, qui questionnent très souvent la forme scolaire traditionnelle (Barrère, 2013). C'est le cas du dispositif MLDS, qui cible les jeunes âgés de plus de 16 ans ayant quitté le système de formation sans obtenir un diplôme de niveau $\mathrm{V}^{9}$ ou plus (Bernard, op. cit.).

Le fonctionnement de la MLDS est relativement autonome. Il rejoint l'idée selon laquelle certains dispositifs de l'institution se distinguent de la forme scolaire traditionnelle par un travail sur la socialisation des individus visant à stimuler la confiance en soi, l' " ouverture d'esprit » ou encore la volonté de jeunes dont les normes et les valeurs seraient préjudiciables à la création d'un projet professionnel, à l'instar de ce qui a été observé dans les dispositifs relais ${ }^{10}$ (Bonnéry \& Martin, 2002 ; Henri-Panabière, Renard \& Thin, 2013 ; Bonnéry \& Renard, 2013).

Après avoir montré que ce travail de socialisation est visiblement assumé dans le dispositif étudié, nous examinerons les réactions qu'il entraîne chez les jeunes accompagnés.

\subsection{Un travail assumé de socialisation}

Les chercheurs travaillant sur les classes relais insistaient sur la tension qui existait, chez les encadrants, entre le rôle d'éducateur et d'enseignant, entre la volonté de socialiser et de transmettre des connaissances (Bonnéry \& Martin, op. cit.). Néanmoins, dans le dispositif MLDS, cette tension est moindre, et les enseignements généraux occupent ici une place assez limitée.

9. CAP (Certificat d'aptitude professionnelle), BEP (Brevet d'études professionnelles) ou DNB (Diplôme nationale du brevet)

10. À destination des collégiens, ces dispositifs accueillent temporairement des élèves en voie de déscolarisation. 


\section{Encadré 2. Les emplois du temps des classes MLDS}

\section{Emploi du temps de la classe MLDS du lycée Jules Vallès}

\begin{tabular}{|c|c|c|c|c|c|}
\hline & LUNDI & MARDI & MERCREDI & JEUDI & VENDREDI \\
\hline $8 \mathrm{H}-8 \mathrm{H} 55$ & & \multirow[b]{2}{*}{ Français } & \multirow[b]{2}{*}{ Sociologie } & \multirow[b]{2}{*}{ Histoire-Géographie } & \\
\hline $8 \mathrm{H} 55-9 \mathrm{H} 50$ & & & & & \\
\hline $\begin{array}{l}10 \mathrm{H} 10- \\
11 \mathrm{H} 05\end{array}$ & \multirow{2}{*}{$\begin{array}{c}\text { TRE } \\
\text { Technique de } \\
\text { recherche d'emploi } \\
\text { et stage }\end{array}$} & \multirow{2}{*}{ Atelier Radio } & \multirow{2}{*}{ Relaxation } & \multirow{2}{*}{ Théâtre } & \\
\hline $\begin{array}{l}11 \mathrm{H} 10- \\
12 \mathrm{H} 05\end{array}$ & & & & & \\
\hline \multicolumn{6}{|l|}{ Déjeuner } \\
\hline $\begin{array}{l}13 \mathrm{H} 30- \\
14 \mathrm{H} 30\end{array}$ & \multirow{2}{*}{ Multimédia } & \multirow{2}{*}{$\begin{array}{c}\text { PSE } \\
\text { Prévention Santé } \\
\text { Environnement }\end{array}$} & \multirow{2}{*}{ Mathématiques } & \multirow{2}{*}{$\begin{array}{l}\text { Connaissance du } \\
\text { monde professionnel }\end{array}$} & \\
\hline $\begin{array}{l}14 \mathrm{H} 30- \\
15 \mathrm{H} 30\end{array}$ & & & & & \\
\hline $\begin{array}{l}15 \mathrm{H} 35- \\
16 \mathrm{H} 30\end{array}$ & & & & & \\
\hline $\begin{array}{l}16 \mathrm{H} 30- \\
17 \mathrm{H} 25\end{array}$ & & & & & \\
\hline
\end{tabular}

Emploi du temps de la classe MLDS du lycée Olympe de Gouges

\begin{tabular}{|c|c|c|c|c|c|}
\hline & LUNDI & MARDI & MERCREDI & JEUDI & VENDREDI \\
\hline \multicolumn{6}{|l|}{$8 \mathrm{H}-8 \mathrm{H} 55$} \\
\hline $8 \mathrm{H} 55-9 \mathrm{H} 55$ & Suivi MLDS & Anglais & & \multirow[b]{2}{*}{ Culture générale } & \\
\hline $\begin{array}{l}10 \mathrm{H} 10-11 \mathrm{H} 05 \\
11 \mathrm{H} 05-12 \mathrm{H} 05\end{array}$ & Atelier Compétences & Atelier Théâtre & Mathématiques & & \\
\hline \multicolumn{6}{|l|}{ Déjeuner } \\
\hline 13H2O-14H15 & Anglais & Suivi MLDS & \multirow{2}{*}{ Atelier expression } & & \\
\hline 14H15-15H15 & \multirow{3}{*}{ Atelier TRE } & & & & \\
\hline $15 \mathrm{H} 30-16 \mathrm{H} 30$ & & Suivi MLDS & & & \\
\hline 16H30-17H25 & & & & & \\
\hline
\end{tabular}

Si les emplois du temps changent au cours de l'année dans les classes MLDS, ils tendent à se stabiliser en milieu d'année, puis à s'appauvrir en fin d'année. Ces deux emplois du temps correspondent alors à ceux fixés en milieu d'année 2016-2017. Les élèves disposent d'un faible volume horaire d'enseignement, 22 heures pour le premier dispositif et 17 heures pour le second. Les enseignements généraux (français, histoire-géographie, anglais, culture générale) représentent moins de la moitié des cours dispensés. L'anglais est la seule langue vivante enseignée, et elle n'existe pas dans le dispositif de Jules Vallès.

L'accent est mis ici sur les enseignements liés aux compétences professionnelles, mais également et surtout sur la sensibilité et la créativité artistique. Outre les ateliers ici présents, en l'occurrence théâtre, radio, relaxation, expression (dans une certaine mesure), les jeunes 
auront participé, durant l'année 2015-2016 et 2016-2017, à ceux de slam, conte, rap et court-métrage.

La prégnance du travail de socialisation dans les classes MLDS pourrait s'expliquer, notamment, par le fait que les jeunes accompagnés ont 16 ans ou plus et sont souvent amenés à se diriger vers des formations professionnalisantes courtes, voire à entrer sur le marché du travail. Cette remise en confiance et ce travail sur l'ouverture aux autres et au monde sont en effet vus par les acteurs comme des moyens de s'adapter à certaines exigences du marché du travail. D'ailleurs, ce travail de socialisation secondaire n'est pas seulement associé à des ateliers psychologiques et artistiques, comme dans les classes relais (Bonnéry \& Martin, 2002). Il s'appuie également ici sur des séances de coaching qui peuvent représenter une part importante du volume horaire, puisqu'elles sont intégrées aux ateliers de techniques de recherche d'emploi et aux cours de connaissance du monde professionnel :

"Moi je fais venir un entrepreneur, un jeune qui vient des quartiers aussi, il les booste vachement. Au début de l'année, il y avait également une chef d'entreprise. Alors tu verrais le choc. Elle les prend en entretien individuel. Elle veut les booster!» (Khadija, 38 ans, coordinatrice MLDS à Jules Vallès)

Cette mise en action de l'élève devrait passer, notamment, par la perception de ses goûts, qu'il devra mettre en lien avec un projet professionnel. Ainsi, lorsqu'au sein de l'établissement où nous travaillions en tant qu'assistant pédagogique ${ }^{11}$, nous avons soumis notre proposition de créer un cours de sociologie, cette discipline semblait être perçue par la coordinatrice comme centrée sur l'individu. Notre cours de sociologie, donné en 20142015, fut ainsi renommé auprès de l'administration, par la coordinatrice, en " atelier d'éducation socio-culturelle " :

"J'aimerais que tu fasses un peu d'orientation avec eux, les faire bosser sur la méthode ADVP (activation du développement vocationnel et personnel), c'est une méthode d'orientation, l'idée c'est de sintéresser d'abord aux goûts des jeunes avant de leur proposer une orientation. " (Mathilde, 36 ans, ancienne coordinatrice MLDS à Jules Vallès)

Ce rôle assumé de socialisation est aussi probablement facilité par la présence réduite d'enseignants au sein du dispositif, dont la routine professionnelle pourrait mettre l'accent sur les savoirs scolaires. En effet, les ateliers professionnalisants, artistiques et culturels, qui représentent environ la moitié du volume horaire de l'emploi du temps des élèves, sont généralement encadrés par des personnes n’appartenant pas au corps enseignant, soit des artistes de diverss horizons, assistants pédagogiques, CPE (Conseiller principal d'éducation), entrepreneurs et même une animatrice de chaîne Youtube. De même, les plateformes de soutien et d'appui aux décrocheurs font intervenir des coordinateurs MLDS, assistants sociaux, responsables d'association, élus locaux, mais très peu d'enseignants. Sur

11. L'établissement Jules Vallès. 
une moyenne de 40 personnes présentes à ces réunions, il n'y aura jamais plus de quatre enseignants.

"Si vous êtes profs, vous n'êtes pas forcément obligés de faire votre matière, vous pouvez avoir une passion, artistique, par exemple, et vouloir la partager. Si vous pensez pouvoir redonner confiance en eux aux élèves en leur proposant de faire d'autres choses avec eux, des activités artistiques. " (Khadija, coordinatrice MLDS cherchant à recruter des intervenants pour le dispositif lors d'une réunion de rentrée de l'établissement Jules Vallès)

Ainsi, le rôle la transmission des savoirs scolaires semble secondaire dans les dispositifs. Les enseignants sont alors appelés non pas à dispenser des cours, mais à transmettre une passion au travers de laquelle les élèves pourraient aussi s'épanouir.

\subsection{Un chahut orienté vers les enseignements les moins scolaires}

Ce travail approfondi de socialisation se heurte à une certaine réticence d'une partie non négligeable des jeunes accompagnés. Les dispositifs sont en effet marqués par un absentéisme important et un chahut particulièrement prégnant au sein de certains enseignements.

Les réticences suscitées par les contenus les moins scolaires des dispositifs de l'Éducation nationale ont été déjà observées, notamment dans les dispositifs relais (Kherroubi, Millet \& Thin, 2015). Il nous semble intéressant ici d'étudier ces réticences par le prisme interactionniste du chahut orchestré par les jeunes accompagnés. Si ce type d'évènements pourrait sembler banal au sein d'un établissement au recrutement social populaire (Testanière, op. cit.; Willis, 1978), et a fortiori dans des dispositifs s'adressant aux jeunes décrocheurs, les séances dans lesquelles ce chahut est particulièrement exacerbé, la forme qu'il prend et les justifications qui en sont données par les élèves permettront de ne pas cantonner l'analyse de ce chahut à une forme de distance des jeunes vis-à-vis de l'institution scolaire.

Il existe des formes d'appropriation des ateliers par les élèves. Certains d'entre eux peuvent participer pleinement à leur bon déroulement, tel cet atelier au sein duquel la réalisation d'un clip de rap a été assez bien accueillie, durant ma première année d'enquête, par la majorité des jeunes, dont deux figures charismatiques de la classe pratiquaient l'écriture amateur de textes. Cette appropriation nécessiterait d'ailleurs une analyse assez fine des formes d'engagement des élèves dans ces ateliers.

Néanmoins, il reste que, de l'aveu même des coordinatrices des classes MLDS, le chahut semble particulièrement problématique au sein des ateliers les plus éloignés de la forme scolaire. Les récits de chahut sont nombreux, parfois violents. Ils sont le plus souvent anodins, mais viennent très fréquemment perturber le déroulement de nombreux ateliers :

"Aussi je travaille beaucoup sur la respiration, avec des éléments de yoga notamment. Fermer les yeux par exemple, alors ça c'est très dur pour eux. A un moment donné, jai enlevé les tables et les chaises. Et je les ai même fait asseoir sur les tables. Cette ouverture, ça ne leur plait pas tou- 
jours. (...) Hier, on a fait massage, enfin du dos, travailler sur les tensions, etc. J'entends 'on est pas pédé.' C'était sûr que ça allait venir ce truc. Et je les ai fait travailler sur un auteur, Tennessee Williams, et je finis par leur dire à la fin qu'il est homosexuel, je l'ai fait exprès. Alors là, c'était 'homo gna gna'» (Emma, 58 ans, assistante pédagogique et encadrante d'un atelier théâtre et philosophie).

De plus, selon une CPE s'occupant des absences des jeunes dans le dispositif de Jules Vallès, ce sont d'ailleurs les ateliers artistiques et culturels qui sont le plus souvent "désertés" bien avant la fin de l'année scolaire, tandis que les cours qui portent sur des matières générales "tiennent mieux le choc".

De nombreux élèves, parfois pour se justifier du chahut ou de leurs absences répétées, soit de manière spontanée en classe, soit lors des entretiens ou des convocations avec les coordinateurs, se montrent très critiques à l'égard de certains ateliers. Leurs reproches visent principalement l'inutilité et la trop grande originalité de ces derniers. La nature des ateliers dépréciés est pourtant très diverse, allant de l'atelier conte à l'atelier slam, en passant par les séances de coaching. Ces récriminations ont émergé très souvent, que ce soit lors des entretiens, au sein d'ateliers ou de cours :

"Genre le lundi y'a un gars y vient pour nous faire faire un clip. Atelier Slam. C'est quoi ça? Ça sert à rien, moi je veux pas mafficher (rires.) On apprend si, on apprend ça, mais on a aucun bulletin, pas de notes. " (Kumba, lors d'un entretien informel)

\section{Encadré 3. Un atelier subverti}

Proposé à certains élèves, cet atelier hebdomadaire d'une heure est encadré par deux assistants d'éducation(AED) qui préparent par ailleurs une licence en psychologie. II consiste à faire parler les élèves d'eux-mêmes, de leur classe, voire de l'actualité. Parfois, un travail rédigé à la maison est exigé. L'exercice à rendre ce jour-là consistait à raconter comment l'élève se voyait dans dix ans. Parmi les trois élèves présents, Sylvain, Sarah et Bakari, seul le troisième avait préparé le travail demandé. Ne prenant pas l'exercice très au sérieux, Bakari fit un récit aventureux avec super héros, combats épiques, explosions et autres incendies, ce qui provoqua le rire de l'assemblée. Sylvain, quelque peu agité, fit également preuve d'humour face aux questions d'un des deux AED venant interrompre l'histoire de Bakari :

"AED: En cours, comment ça va?

Sylvain : Je rigole un peu, c'est tout. Qu'est-ce que j'ai fait pour être là ? Je tiens à dire que j'ai pas fait preuve d'insolence!

AED: Un de tes profs m'a dit « Ah Sylvain, il est sympa, mais il est un peu lourd. »

S. : lls sont jaloux c'est tout. 
AED: Ton comportement, c'est un peu exubérant non?

$S$. : Je suis un enfant.

AED : $A$ 17 ans ?

S. : Oui et même après, ma femme elle sera avec un enfant.

$A E D$ : Et pourquoi tu veux faire l'enfant?

S. : Allez, c'est quoi ces questions?

AED : Donne un argument.

S. : (après un moment de silence) Je passe mon tour.

AED : On vient te revoir après.

Bakari : Bon, je termine mon histoire. Vous voyez ce petit papier (il lève la feuille sur laquelle est écrit son récit). Bah, il va vous faire kiffer!»

Bakari continue son histoire rocambolesque, tandis que Sylvain l'interrompt alors qu'il narrait une scène dans laquelle il combattait un ennemi, juché à la verticale sur un gratte-ciel.

"S. (impatienté) : Ouah, mais c'est quoi ce truc de shlag ici! (expression polysémique désignant notamment une personne ou une chose associée à l'étrangeté ou à la pauvreté)

AED : (à Bakari) La réalité, tu l'as mise de côté ?

B. : Oui, car elle est moche!»

Les questions des encadrants, comme l'exercice qu'ils ont proposé, visent à produire un discours sur soi, mais également à développer un regard analytique sur ses actions ou ses propos : "La réalité tu l'as mise de côté?" ou encore "et pourquoi tu veux faire l'enfant?". Si l'exercice proposé semble assez peu étayé, les finalités de l'atelier semblent bien comprises par Sylvain, qui ne semble pas être victime d'un "malentendu socio-cognitif ${ }^{2}$ ". En effet, chacune de ses réponses, données le plus souvent avec un air sarcastique, prennent volontairement le contrepied des propos de l'apprenti psychologue. Loin de révéler une incapacité à faire preuve de réflexivité sur son comportement, ses réponses ironiques montrent, en négatif, que Sylvain connait les attentes de l'encadrant. Les formes prises par ce chahut, celles des refus systématiques, le fait de tourner en dérision des questions et l'exercice pourraient tenir à une forme de réticence au travail de socialisation. Pour Bakari, il pourrait même s'agir de " rescolariser" l'exercice en transformant un récit analytique sur soi en travail scolaire ordinaire d'écriture et d'imagination.

Ce travail de socialisation se heurte donc à certaines réticences, qui passent notamment par un chahut qui, souvent orienté vers les dimensions les plus éloignées des enseignements ordinaires, prend souvent la forme du refus systématique et le fait de tourner en dérision. Nous nous interrogerons dans la partie suivante sur le sens que peut recouvrir ces réactions.

12. Les malentendus socio-cognitifs désignent des situations d'apprentissage dans lesquelles la volonté de construire le savoir à partir des élèves participe de l'invisibilisation des finalités réelles de l'exercice (Bautier \& Rayou, 2009.) 


\section{Le sens
des réticences}

Le chahut est généralement analysé comme le résultat d'une distance, voire d'une contradiction entre les logiques socialisatrices de la famille ou du groupe de pairs des élèves avec celle de l'École. Selon Jacques Testanière (op. cit.), le chahut traditionnel, comme le bizutage, consistait en une forme de désordre encadré et systématique, faisant office de rite d'initiation exprimant et renforçant l'intégration. Il aurait été substitué, avec la massification du niveau secondaire, au chahut anomique, vu comme un désordre généralisé nuisant à la transmission pédagogique et témoignant d'un manque d'intégration des élèves aux objectifs institutionnels. Paul Willis (op. cit.), ou plus récemment Thomas Sauvadet (2006), voient dans le chahut, pour l'un, la volonté de s'affirmer en tant que membre de la classe ouvrière, avec des attitudes viriles s'opposant à celles imposées par la forme scolaire, et pour l'autre, la nécessité, pour les jeunes des classes populaires, d'exhiber devant leurs pairs la détention d'un capital guerrier, une " combinaison de force physique, de techniques, de dispositions psychosociologiques et de relations sociales, orientée vers l'aptitude au combat et assurant une temporaire et relative sécurité aux niveaux symbolique, économique et physique.»

Dans cette seconde partie, nous verrons d'abord que le chahut mis en place ne pourrait être qualifié d'anomique ou du moins être analysé qu'en termes de distance des jeunes vis-à-vis l'institution scolaire et, ensuite, qu'il pourrait même s'apparenter à une forme de policy feedback (Dupuy \& Halpern, 2009), c'est-à-dire comme la protestation plus ou moins organisée d'un groupe visé par une politique publique.

\subsection{Une volonté de normalité ?}

Le stigmate assigné aux décrocheurs a été principalement analysé sous trois angles. D'abord, des recherches se sont intéressées à celui provoqué par la carrière scolaire des décrocheurs, en montrant que cette dernière, souvent ponctuée de nombreux échecs scolaires et d'un jugement négatif de l'institution, s'apparente à "un parcours de disqualification symbolique" (Millet \& Thin, 2004). Cet étiquetage déviant des décrocheurs a également été corrélé avec l'autonomie des dispositifs par rapport aux établissements scolaires, en raison de la séparation ainsi créée entre les élèves ordinaires et les décrocheurs (Bonnéry \& Martin, 2002). Enfin, en adoptant un point de vue plus macrosocial, Bertrand Geay (2003) montre que le développement des politiques de lutte contre les violences et l'absentéisme, depuis les années 1980, a substitué à l'image du "cancre ", "chahuteur aux allures sympathiques" assis au fond de la classe, celle du "sauvageon ${ }^{13}$ " qui, ne pouvant s'insérer sur le marché du travail sans diplômes, risquerait de troubler l'ordre social. Ce qui nous intéressera ici est l'effet propre de ce travail de socialisation sur le sentiment de stigmatisation exprimé par les décrocheurs accompagnés.

13. Expression utilisée par l'ancien ministre de l'Éducation nationale, Jean-Pierre Chevènement. 
Les classes MLDS veulent pourtant éviter la stigmatisation des décrocheurs. Elles tentent même souvent d'y apporter des réponses. En effet, comme nous l'avons vu plus haut, l'encadrement des jeunes vise notamment à leur redonner confiance, les classes MLDS sont généralement accueillies au sein des établissements scolaires ordinaires et les acteurs insistent souvent sur la nécessité de faire preuve d'une certaine bienveillance dont manquerait l'institution scolaire.

\section{Encadré 4 : Une salle siglée « MLDS" (Mission de lutte contre le décrochage scolaire)}

À Jules Vallès, un écriteau sur lequel est inscrit « MLDS » est fixé sur la porte de la salle de classe. Si les élèves de l'établissement, y compris du dispositif, ne connaissent pas forcément la signification de cet acronyme, il reste que celui-ci est assimilé à une classe anormale. Quand des élèves des filières ordinaires de l'établissement qui nous connaissaient ont appris que nous travaillions dans cette classe, certains ont formulé des remarques négatives au sujet de celle-ci. «Ah ouais, maintenant genre tu bosses avec les cassos » (diminutif de " cas sociaux »). Le sentiment de stigmate, au moins à l'intérieur de l'établissement, s'appuie donc sur une réalité vécue, notamment dans les interactions avec les autres élèves, comme celle que nous avons pu observer durant un intercours. Stéphane se balade prêt de la porte de la salle lorsqu'il croise un ancien compagnon de classe qui passe dans le couloir :

"Camarade: Ah l'ancien! Qu'est-ce tu fais là?

Stéphane: Bah vas-y, je me suis fait virer de mon ancien lycée!

C. : Non, pourquoi ?

S. : J'ai tapé un bouffon (rires du camarade, mais Stéphane semble gêné)

C. : "Et t'es en quelle classe genre?

S. : (se gratte la tête) Bah le truc là... MLDS

C. : Non!»

Plusieurs élèves ont mis en avant l'aspect stigmatisant de cet écriteau collé à la porte de la salle de classe. Ces remarques ont fait débat au sein de la réunion de concertation. En effet, cette observation faisait consensus parmi les encadrants de la classe MLDS. Néanmoins, tous n'étaient pas d'accord s'agissant des solutions à apporter. Durant leur cours, les enseignantes de français-histoire-géographie avaient alors distribué des questionnaires aux élèves pour savoir s'ils voulaient retirer le sigle de la porte, le garder ou le recouvrir avec un poster, par exemple. C'est cette dernière solution qui a été choisie à la quasi-unanimité des élèves.

Néanmoins, les spécificités du chahut ayant cours dans les dispositifs, comme les justifications que les élèves en apportent, interrogent sur l'efficacité de ces réponses à la stigmatisation des décrocheurs.

En effet, il semble que ce travail sur la confiance en soi soit justement source, pour les jeunes des dispositifs, d'un sentiment d'être traités différemment des autres élèves ; comme des " collégiens", des "SEGPA" ", avec des ateliers "chelous", et des cours sans "vraies

14. Dans le discours des élèves, l'acronyme semble être ainsi devenu une insulte. Une série humoristique 
notes ", puisque les enseignants "not(erai)ent trop bien" (termes issus d'une discussion collective avec des élèves du dispositif de Jules Vallès.)

Ce rejet est généralement justifié par une revendication de normalité : "Moi je veux des vrais cours, avec du vrai français, des vrais maths, du vrai anglais. " (Jonathan) ou encore "vas-y, on fait des trucs de SEGPA " (Bintou). Parfois, leurs critiques n'épargnent guère les matières générales, mais questionnent surtout le niveau du contenu et les modalités de notation au sein du dispositif. Certains élèves diront, comme Nicolas : "Non mais franchement, en math, ce qu'on fait, c'est niveau collège" ou encore Jennifer : "Ici c'est trop facile, vous notez trop bien " et Kumba : "Attendez, avant, dans ma classe, j'étais un des plus mauvais, ici je suis un des meilleurs!".

Compte tenu de ces discours, le chahut mis en place par les jeunes ne pourrait pas seulement être interprété, comme chez Paul Willis (op. cit.) ou Thomas Sauvadet (op. cit.), comme une opposition à la norme scolaire pour affirmer une identité sociale d'ouvrier ou de jeune des classes populaires. Ici, il vient au contraire justifier la norme scolaire en s'en prenant à l'originalité des dispositifs pour défendre leur normalité d'élève.

Lors des entretiens, lorsque nous avons posé la question "Qu'est-ce que tu proposerais d'ajouter aux classes MLDS pour améliorer leur fonctionnement?" aux jeunes du dispositif, sept des onze élèves interrogés évoquaient l'ajout de cours d'anglais. Une matière générale qui manquait en effet au programme de cette classe MLDS. La coordinatrice du dispositif d'Olympe de Gouges semble d'ailleurs avoir bien conscience que l'originalité des ateliers se heurte à des réticences de la part des jeunes accompagnés :

"En classe, si tu proposes des trucs un peu trop différents et alternatifs, les gamins vont te demander des cours plus classiques, de faire de la grammaire et de la conjugaison, faire des exercices, alors quills détestaient ça à l'école. " (Lauryne, 35 ans, coordinatrice MLDS du lycée Olympe de Gouges)

\subsection{Un refus d'obtempérer aux nouvelles formes d'accompagnement social?}

La plupart des jeunes accompagnés ne rejettent pas totalement le dispositif. Certains reconnaissent notamment la bienveillance des encadrants. "Ici, faut dire quand même que quand t'as besoin d'eux, ils t'aident "s'exclame ainsi Kevin, alors qu'une discussion à plusieurs remettait en cause le dispositif, propos auxquels ses camarades semblaient acquiescer. Cette ambivalence à l'égard de certains dispositifs scolaires de seconde chance avait d'ailleurs été analysée s'agissant des parents d'élèves scolarisés en classes relais (Millet \& Thin, op. cit.).

éponyme, mettant en scène une classe SEGPA, totalise d'ailleurs plusieurs millions de vues sur Youtube. 
Néanmoins, les griefs visent peut-être la forme même prise par cette aide lorsqu'on la met en relation avec les changements intervenus dans les politiques sociales et les représentations sur les individus aidés. En effet, le curriculum caché ${ }^{15}$ de ces dispositifs pourrait résider dans l'assignation du stigmate à l'individu accompagné, mais également dans la responsabilisation individuelle de l'échec scolaire.

Si le décrochage scolaire est "le nouveau nom donné à l'échec scolaire" (Glasman, 2012), il pourrait également exacerber les nouvelles manières d'appréhender l'échec scolaire. L'idée que les décrocheurs manqueraient de confiance en eux rappelle ainsi la médicalisation de l'échec scolaire. Particulièrement prégnante depuis le milieu des années 1990, elle associe les difficultés scolaires à des causes psychologiques, voire biologiques, et propose d'y remédier par un accompagnement, voire un traitement ad hoc (Morel, 2014). De même, la volonté de " resocialiser " les décrocheurs, en travaillant notamment sur leur ouverture d'esprit, coïncide avec la thématique du " handicap socio-culturel », vulgarisation déformée des théories sociologiques des inégalités scolaires des années 1960 consistant à interpréter l'écart culturel entre les élèves issus des familles populaires et l'école comme un manque et une forme de fermeture culturelle (Thin, 1998).

"On va l'appeler Anne. 14 ans et demi. Mère totalement injoignable. Père maltraitant avec son épouse :

"Je suis la mère de ma mère, moi à 5 ans, quand je voulais manger, il fallait que je me fasse à manger".

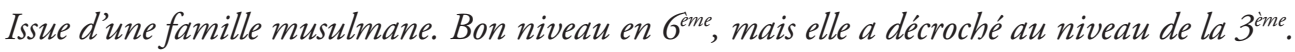
La mère a des problèmes psys. Elle a une sorte de beau-père:

"À 12 ans, j’ai connu l'alcool, la drogue, mais ça c’était avant".

A eu une grossesse. Les AS sinquiètent de son psychisme, elle manquait beaucoup de motivation. Et bien on a été nombreux à l'aider sur le territoire et sa situation s'est améliorée (...) Aujourd'hui, elle a 14 de moyenne on lui a trouvé une filière et un stage. " (Marine, 50 ans, assistante sociale, présentant le cas d'une adolescente décrocheuse lors d'une réunion de la PSAD)».

Ce récits sur les élèves, émanant des acteurs des dispositifs, sont assez communs. Pourtant, ils pourraient difficilement être généralisés à l'ensemble des jeunes ayant quitté l'école avant d'y obtenir un diplôme. La construction formelle du récit de l'assistante sociale est d'ailleurs assez intriguante. Les phrases courtes, souvent privées de verbes, semblent symboliser l'addition sans fin d'évènements et de caractéristiques défavorables. Les citations marquantes viennent ajouter à la véracité du récit en faisant dire à l'adolescente les faits les plus tragiques notamment : " $\grave{A} 12$ ans, j’ai connu l'alcool, la drogue (...). » Néanmoins, ces

15. Le curriculum caché désigne la part des apprentissages qui n’est pas programmée explicitement dans l'institution scolaire et qui peut avoir des effets inattendus (Perrenoud, 1993). 
cas difficiles, surtout s'ils sont en partie résolus par l'intermédiaire des dispositifs, fonctionnent comme une forme de récit auto-justificateur, venant à la fois légitimer l'action des dispositifs et le regard que les accompagnateurs portent sur les jeunes accompagnés.

Ces tendances à appréhender le décrochage sous l'angle de la psychologie ou du handicap socio-culturel pourraient aussi concourir à une logique de responsabilisation individuelle et familiale du décrochage scolaire :

"Comment arriver à leur montrer qu'ily a d'autres métiers possibles? Où il y a plus de places. Le champ des possibles, ouvrir leur esprit. En fait, leurs représentations, c'est les métiers des copains, des grands frères, leurs papa-maman." (Lauryne, 35 ans, coordinatrice MLDS du lycée Olympe de Gouges)

Par un subtil jeu d'inversion, les discours des acteurs sur le manque d'ouverture d'esprit des jeunes appréhendent la pénurie d'emplois comme un problème moins lié à son offre qu'à sa demande. Si les jeunes en question faisaient preuve d'ouverture d'esprit, ils sauraient alors s'orienter vers des filières d'avenir susceptibles d'offrir une formation et un emploi stable. Cette responsabilisation individuelle de la situation des jeunes non-diplômés rejoint ainsi celle des " assistés », dont les difficultés se retrouvent parfois naturalisées (Bodin, 2012) et dont la volonté de trouver un emploi doit être surveillée et contrôlée (Dubois, 2007). Une logique qui individualise, voire essentialise les difficultés sociales des individus plutôt que de chercher à questionner les structures éducatives ou économiques (Castel \& Le Cerf, 1980 ; Neveu, 2015).

C'est aussi la forme prise par les interactions avec les coordinateurs et les différents acteurs du dispositif qui présentent des affinités électives avec les nouvelles politiques sociales. La " matrice contractuelle " et la conditionnalisation croissante des aides au sein de ces politiques visent à exiger de l'individu aidé un certain nombre de contreparties (Castel, op. cit ; Dufour \& al., 2008). Ces exigences se focalisent souvent sur la démarche d'insertion de l'individu aidé. Elle peut revêtir plusieurs formes dans les dispositifs. Tout d'abord, les places vacantes y sont limitées et l'entrée n'y est pas toujours aisée. Les jeunes peuvent ainsi passer une série d'entretiens au cours desquels ils doivent s'engager, parfois sous forme de contrat, à répondre aux attentes du dispositif. Ensuite, les élèves peuvent également réintégrer des filières ordinaires durant l'année en cours ou l'année suivante, souhaits pour lesquels l'appui du coordinateur pourra être utile, voire nécessaire. À la manière de l'intervenant social qui est ainsi érigé en juge de la légitimité de la demande et accorde ou non la prestation financière en fonction de cette évaluation (Castel \& Haroche, op. cit.), une coordinatrice du dispositif se montre parfois mal à l'aise avec cette forme de "magistrature morale " exercée sur les jeunes accompagnés :

"Une fois, j’ai eu une gamine qui était là. Elle ne voulait absolument pas sinscrire en $M L D S$. Elle voulait trouver un lycée professionnel, elle n'avait pas eu son affectation. Et bien la gamine elle est venue sept fois! Sept fois! Pour me le dire. Moi je me suis dit, faut pas le prendre personnellement, et elle m'a touchée, alors j'ai fait les choses pour qu'elle trouve une 
affectation. Mais si un autre ne me touche pas? Qui on est pour juger de ces gamins-là? Pour choisir de donner sa chance à l'un et pas à l'autre?" (Khadija, coordinatrice MLDS à Jules Vallès)

Si, à la manière de l'élève dont parle Khadija, certains jeunes accompagnés essayent, au cours de l'année, voire comme ici avant même leur scolarisation en classe MLDS, de regagner une place dans les filières ordinaires ${ }^{16}$, au départ, ils cherchent tout de même à intégrer les dispositifs. Une inscription qui pourra leur permettre d'obtenir une éventuelle formation, de faciliter leur reprise d'études ou simplement de s'occuper et se défendre d'être inactifs. Répondant difficilement aux exigences des dispositifs et n'en comprenant pas souvent les enjeux, ils n’ont d'autres choix, comme le bénéficiaire d'aide chez Castel (op. cit.), de construire un récit auto-justificateur impliquant des difficultés scolaires insurmontables ou des difficultés sociales empêchant leur implication. En « touchant » ainsi le coordinateur, ils peuvent s'assurer de son soutien, mais valident ainsi les représentations misérabilistes sur les décrocheurs.

De même, le chahut orienté vers les écarts faits à la norme scolaire peut aussi paradoxalement venir légitimer à la fois le stigmate associé aux décrocheurs et le travail de socialisation sur la confiance en soi, l'ouverture d'esprit et la volonté de ces derniers. Une intervenante responsable d'un atelier théâtre dira ainsi : "Je leur fais faire des exercices où ils doivent simplement toucher l'épaule de l'autre, et ils le prennent super mal, ils disent que c'est trop bizarre. "Paroles qui pourront prouver ainsi, à ses yeux, la fermeture d'esprit de ces jeunes et donc l'utilité de travailler sur cet aspect. De même, un intervenant menant un atelier radio me confiera : "Et là ils me demandent" non mais ça sert à quoi de faire ça?". Ils sintéressent à rien, ils restent assis sur leurs chaises comme des loques. " D'où l'intérêt qu'il y aurait à travailler sur leur volonté.

On pourrait ainsi comparer ce chahut à une forme de protestation de policy outsiders, soit d'un groupe sans ressources politiques (Dupuy \& Halpern, op. cit.), ne pouvant exprimer leur mécontentement que par ce biais. Loin d'infléchir la politique en question, cette protestation viendrait alors, au contraire, la renforcer, en légitimant le mode d'encadrement mis en place.

\section{Conclusion}

Comme cela a été observé dans les classes relais (Kherroubi, Millet \& Thin, op. cit.), notre enquête montre que les actions du dispositif MLDS (Mission de lutte contre le décrochage scolaire) se heurtent à de nombreuses réticences de la part des individus accompagnés. Nous nous sommes focalisés ici non pas sur les acteurs des dispositifs ou

16. Ce qui, pour certains des coordinateurs, dont Mathilde (ancienne coordinatrice à Jules Vallès), a comme effet pervers de générer une déperdition des "meilleurs éléments" au cours de l'année. 
les parents des jeunes accompagnés (Millet \& Thin, op. cit.), mais sur les principaux intéressés, notamment dans leurs interactions quotidiennes avec le dispositif.

Nous avons alors étudié leurs réticences par le prisme du chahut qui, loin de n'être qu'anomique ou du moins opposé au fonctionnement de l'institution (Testanière, $o p$. cit.; Willis, op. cit. ; Sauvadet, op. cit. ; Barrère, op. cit.), semble servir à la revendication d'une scolarisation ordinaire d'élèves comme les autres. En raison de leur distance visà-vis des normes scolaires ordinaires, certains ateliers et modalités de cours donnent à ces jeunes le sentiment d'être traités trop différemment des autres élèves. Les acteurs des dispositifs prennent pourtant en compte ce sentiment de stigmate. Or, les actions qu'ils entreprennent pour l'éviter, notamment en mettant l'accent sur les enseignements artistiques et culturels visant à travailler sur la confiance en soi, favorisent paradoxalement cette impression.

Le mode d'encadrement mis en place, qui a été ici mis en perspective avec les nouvelles manières de se représenter et d'agir sur les difficultés sociales (Castel, op. cit. ; Castel \& Haroche, op. cit. ; Dubois, op. cit. ; Dufour \& al., 2008 ; Bodin, op. cit.), tend à imputer aux individus et à leur cercle social proche les causes de leur situation. En pointant du doigt un manque de confiance en soi, d'ouverture et de motivation par le biais du registre "psy" (Morel, op. cit.), de la thématique du handicap socio-culturel (Thin, $o p$. cit.) et d'une vision naïvement idéologique du marché du travail, ce dispositif issu d'une nouvelle politique éducative peut participer, à l'instar des nouvelles politiques sociales, de la responsabilisation individuelle des problèmes sociaux et de l'assignation d'un stigmate aux individus aidés. Le chahut des jeunes accompagnés, fait de refus systématique et d'ironie, s'en prend à ce qui symbolise le plus ce traitement stigmatisant. Il pourrait être relié à la notion des policy feedbacks, réactions et protestations des groupes concernés par une politique publique, ici sous une forme non légitime de policy outsiders sans ressources politiques (Dupuy \& Halpern, op. cit.).

Si les dispositifs centrés sur la résolution d'un problème particulier et ciblant une population précise se multiplient au sein de l'institution scolaire (Barrère, op. cit.), il reste que cet encadrement différencié peut avoir des effets pervers. La place prépondérante accordée au travail de socialisation pourrait aussi être discutée, non seulement du point de vue de son efficacité, mais aussi de ses effets contextuels qui viennent ici parachever cette mise à l'écart objective et subjective du système scolaire ordinaire. On pourrait ainsi appuyer les préconisations du CNESCO (Conseil national d'évaluation du système scolaire) sur la lutte contre le décrochage scolaire, appelant notamment à travailler davantage en amont du décrochage (Bernard, 2017). 


\section{Bibliographie}

Barrère A. (2002), "Un nouvel âge du désordre scolaire : les enseignants face aux incidents ", Déviance et Société, vol. 26, n 1, pp. 3-19.

Barrère A. (2013), "La montée des dispositifs : un nouvel âge de l'organisation scolaire ", Carrefours de l'éducation, n 36, pp. 95-116.

Bautier E. \& Rayou P. (2009), Les inégalités d'apprentissage, Paris, Presses universitaires de France, 172 p.

Bernard P.-Y. (2011), Le décrochage scolaire, Paris, Presses universitaires de France.

Bernard P.-Y. (2017), Le décrochage scolaire en France : du problème institutionnel aux politiques éducatives, Paris, Cnesco, $76 \mathrm{p}$.

Bonnéry S. \& Renard, F. (2013), «Des pratiques culturelles contre l'échec et le décrochage scolaires. Sociologie d'un détour ", Lien social et Politiques, n 70, pp. 135-150.

Martin E. \& Bonnéry S. (2002), Les classes relais : Un dispositif pour les élèves en rupture avec l'école, Issy-les-Moulineaux, ESF, 212 p.

Blaya C. (2010), Décrochages scolaires. L'école en difficulté, Bruxelles, De Boeck, 200 p.

Bodin R. (2012), "Rationalisation de l'action sociale et naturalisation des inégalités ", in Bodin R., Les métamorphoses du contrôle social, Paris, La Dispute, pp. 103-114.

Bourdieu P. (2012), Sur l'État. Cours au Collège de France 1989-1992, Paris, Seuil et Raisons d'agir, $672 \mathrm{p}$.

Bruneteaux P. \& Lanzarini C. (1998), "Les entretiens informels ", Sociétés contemporaines, vol. $30, \mathrm{n}^{\circ} 1$, pp. 157-180.

Bruno F., Saujat F. \& Félix C. (2015), « Les programmes de prévention et de lutte contre le décrochage scolaire et leurs conséquences sur le travail enseignant : revue de littérature ", Revue française de pédagogie. Recherches en éducation, $\mathrm{n}^{\circ}$ 193, pp. 89-104.

Castel R. (1995), Les métamorphoses de la question sociale : une chronique du salariat, Paris, Fayard, "L'espace du politique », 490 p.

Castel R. \& Cerf J.-F. L. (1980), "Le phénomène 'psy’ et la société française », Le Débat, $\mathrm{n}^{\circ} 1$, pp. 32-45.

Dubois V. (2007), "État social actif et contrôle des chômeurs : un tournant rigoriste entre tendances européennes et logiques nationales ", Politique européenne, n 21, pp. 73-95.

Dufour P., Boismenu G. \& Noël A. (2008), L'aide au conditionnel la contrepartie dans les mesures envers les personnes sans emploi en Europe et en Amérique du Nord, Ed. Les Presses de l'université de Montréal-PIE, Bruxelles, Peter Lang, 25 p. 
Dupuy C. \& Halpern C. (2009), "Les politiques publiques face à leurs protestataires », Revue française de science politique, vol. 59(4), pp. 701-722.

Geay B. (2003), «Du 'cancre' au 'sauvageon' : les conditions institutionnelles de diffusion des politiques d'insertion' et de 'tolérance zéro' ", Actes de la recherche en sciences sociales, vol. ${ }^{\circ} 149, \mathrm{n}^{\circ} 4$, pp. 21-31.

Glasman D. (2012), "Le décrochage scolaire, nouveau nom de l'échec scolaire ", VEI Diversité, Hors-série n ${ }^{\circ} 14$, pp. 7-14.

Henri-Panabière G., Renard F. \& Thin D. (2013), «Des détours pour un retour? Pratiques pédagogiques et socialisatrices en ateliers relais ", Revue française de pédagogie. Recherches en éducation, $\mathrm{n}^{\circ} 183$, pp. 71-82.

INSEE (2013), "Les décrocheurs du système éducatif : de qui parle-t-on ? ", France Portrait social, Insee.

Kherroubi M., Millet M. \& Thin D. (2015), Désordre scolaire: l'école, les familles et les dispositifs relais, Paris, Pétra, "Éducation, art du possible », $336 \mathrm{p}$.

Mauger G. (1991), "Enquêter en milieu populaire », Genèses, vol. 6, n 1, pp. 125-143.

Millet L. et Thin D. (2012), "L'ambivalence des parents de classes populaires à l'égard des institutions de remédiation scolaire. L'exemple des dispositifs relais ", Sociétés contemporaines, $\mathrm{n}^{\circ} 86$, pp. 59-83.

Morel S. (2014), La Médicalisation de l'échec scolaire, Paris, La Dispute, "L'enjeu scolaire ", $210 \mathrm{p}$.

Neveu É. (2015), Sociologie des mouvements sociaux, Paris, La Découverte, 128 p.

Perrenoud P. (1993), "Curriculum : le formel, le réel, le caché », in Houssaye J. (dir.), La pédagogie : une encyclopédie pour aujourd'hui, Paris, ESF, pp. 61-76.

Pothet J. (2016), "Des parents 'défaillants' ", Actes de la recherche en sciences sociales, n ${ }^{\circ} 214$, pp. 66-79.

Sauvadet T. (2006), Le capital guerrier, Concurrence et solidarité entre jeunes de cité, Paris, rmand Colin, "Sociétales », 303 p.

Testanière J. (1967), « Chahut traditionnel et chahut anomique dans l'enseignement du second degré ", Revue française de sociologie, vol. 8, n 1, pp. 17-33.

Thin D. (1998), Quartiers populaires. L'école et les familles, Lyon : PUL.

Willis P. (1978), "L'école des ouvriers ", Actes de la recherche en sciences sociales, vol. 24, pp. 50-61. 